\title{
Connection between the Star Formation Rate and the Gamma-Ray Bursts
}

\author{
Attila Mészáros ${ }^{1}$, Zsolt Bagoly ${ }^{2}$, Lajos G. Balázs ${ }^{3}$ and \\ István Horváth ${ }^{4}$ \\ ${ }^{1}$ Faculty of Mathematics and Physics, Astronomical Institute, Charles University, V \\ Holešovičkách 2, CZ 18000 Prague 8, Czech Republic, email: meszaros@cesnet.cz; \\ ${ }^{2}$ Department of Physics of Complex Systems, Eötvös University, H-1117 Budapest, Pázmány \\ P. s. 1/A, Hungary; \\ ${ }^{3}$ Konkoly Observatory, H-1505 Budapest, POB 67, Hungary; \\ ${ }^{4}$ Department of Physics, Bolyai Military University, H-1581 Budapest, POB 15, Hungary
}

\begin{abstract}
It is remarkable that the long gamma-ray bursts, as objects connected with the supernovae - i.e. with the end of the massive stars, trace the star formation rate. This connection is discussed in this contribution. The presentation is in essence a recapitulation of the article Mészáros A. et al. A\&广A, 2006, 455, 785.
\end{abstract}

Keywords. gamma rays: bursts — supernovae: general — stars: formation

\section{Overview of the article Mészáros et al. (2006)}

The BATSE instrument on the Compton Gamma Ray Observatory detected 2704 gamma-ray bursts (GRBs). From this data set it follows that there are two physically different subgroups of GRBs, "short" and "long" ones (Balázs et al. 2003); further subgroups may also exist (see Rípa et al. 2012 and the references therein).

The question is the following: Can the redshifts of GRBs be distributed in accordance with other objects arising in star formation regions (Madau 1995 and Dahlen et al. 2004)? In addition, this question should be answered separately for any subgroup.

The method is the following: We assume for a given subclass of GRBs that it is distributed in accordance with the redshift distribution of the objects in star formation regions. Then we compare this theoretical expectation with the observational data from the BATSE Catalog.

The answer is that the redshift distribution of the long bursts may be proportional to star-formation rate (SFR). For the short bursts this can also happen, but the proportionality is less evident. The connection of the eventual third subgroup and of the SFR was not studied yet. All these results are independent on the models of GRBs, and also on the cosmological parameters.

Acknowledgements. This study was supported by the OTKA grant K77795, by the Grant Agency of the Czech Republic grants No. P209/10/0734, and by the Research Program MSM0021620860 of the Ministry of Education of the Czech Republic.

\section{References}

Balázs, L. G., Bagoly, Z., Horváth, I., Mészáros, A., \& Mészáros, P. 2003, Aछ̛A, 401, 129

Dahlen, T., Strolger, L. G., Riess, A. G., et al. 2004, ApJ, 613, 189

Madau, P. 1995, ApJ, 441, 18

Mészáros, A., Bagoly, Z., Balázs, L. G., \& Horváth, I. 2006, A $\& A$, 455, 785

Rípa, J., Mészáros, A., Veres, P., \& Park, I. H. 2012, ApJ, 756, 44 